\title{
A genetically encoded fluorescent sensor for in vivo imaging of GABA
}

Jonathan S. Marvin ${ }^{1}$, Yoshiteru Shimoda ${ }^{2}$, Vincent Magloire ${ }^{2}$, Marco Leite ${ }^{2}$, Takashi

Kawashima $^{1}$, Thomas P. Jensen ${ }^{2}$, Erika L. Knott ${ }^{3}$, Ondrej Novak ${ }^{1,4}$, Kaspar Podgorski ${ }^{1}$, Nancy J. Leidenheimer ${ }^{3}$, Dmitri A. Rusakov ${ }^{2}$, Misha B. Ahrens ${ }^{1}$, Dimitri M. Kullmann ${ }^{2}$, Loren L. Looger ${ }^{1}$

${ }^{1}$ Janelia Research Campus, Howard Hughes Medical Institute, Ashburn, VA

${ }^{2}$ Institute of Neurology, University College London, UK

${ }^{3}$ Department of Biochemistry \& Molecular Biology, Louisiana State University Health Sciences Center, Shreveport, LA

${ }^{4}$ Department of Auditory Neuroscience, Institute of Experimental Medicine, Academy of Sciences of the Czech Republic, Prague, Czech Republic

Protein engineering and experiment design: JSM, LLL

Hippocampal slice imaging: TPJ, DAR

Visual cortex volume imaging: KP, ON

Mouse epilepsy model: YS, VM, ML, DMK

Mitochondria experiments: ELK, NJL

Zebrafish: TK, MBA 


\begin{abstract}
(150 words)
Current techniques for monitoring GABA, the primary inhibitory neurotransmitter in vertebrates, cannot follow ephemeral transients in intact neural circuits. We applied the design principles used to create iGluSnFR, a fluorescent reporter of synaptic glutamate, to develop a GABA sensor using a protein derived from a previously unsequenced Pseudomonas fluorescens strain. Structure-guided mutagenesis and library screening led to a usable iGABASnFR $\left(\Delta \mathrm{F} / \mathrm{F}_{\max } \sim 2.5\right.$, $\mathrm{K}_{\mathrm{d}} \sim 9 \mu \mathrm{M}$, good specificity, adequate kinetics). iGABASnFR is genetically encoded, detects single action potential-evoked GABA release events in culture, and produces readily detectable fluorescence increases in vivo in mice and zebrafish. iGABASnFR enabled tracking of: (1) mitochondrial GABA content and its modulation by an anticonvulsant; (2) swimming-evoked GABAergic transmission in zebrafish cerebellum; (3) GABA release events during inter-ictal spikes and seizures in awake mice; and (4) GABAergic tone decreases during isoflurane anesthesia. iGABASnFR will permit high spatiotemporal resolution of GABA signaling in intact preparations.
\end{abstract}

Introduction (2500-3500 words for Intro, Results, Discussion)

$\gamma$-Amino butyric acid (GABA) is a ubiquitous inhibitory neurotransmitter (the principal one in vertebrates), reducing neuronal excitability by receptor-mediated hyperpolarization of membrane potential and shunting of excitatory currents. GABA can also be excitatory in early development and in cell types with high intracellular chloride ${ }^{1}$. GABA receptors are hetero-pentameric $\mathrm{Cl}^{-}-$ conducting channels ${ }^{2}$. GABA $\mathrm{B}$ receptors are hetero-dimeric metabotropic receptors that are coupled to multiple ionic currents by $\mathrm{G}_{\mathrm{i} / \mathrm{o}}$-proteins ${ }^{3,4}$, and are more sensitive to GABA than most synaptic GABAA $_{A}$ receptors ${ }^{5}$. The inhibitory role of GABA and its receptors has been the subject of significant pharmacological intervention. Drug classes include GABAA receptor allosteric modulators (e.g. sedative, anxiolytic and anticonvulsant benzodiazepines), GABA $\mathrm{B}$ agonists (e.g. the anti-spastic baclofen), GABA reuptake inhibitors (e.g. the anticonvulsant tiagabine), and GABA transaminase inhibitors (e.g. the antiepileptic vigabatrin). GABA receptor antagonists include both drugs and toxins.

Despite the clinical efficacy of drugs that augment GABAergic neurotransmission, the details of GABA release from neurons, diffusion to both post- and pre-synaptic receptors, reuptake by neurons and astrocytes, recycling through the glutamate/GABA-glutamine shuttle ${ }^{6}$, and other critical mechanisms, including its role in metabolism, remain largely unknown. GABA signaling has traditionally been inferred from a combination of electrophysiology and pharmacology ${ }^{7}$. Direct detection of GABA has usually been achieved by microdialysis followed by derivatization and quantification by HPLC/electrochemical detection ${ }^{8}$. In addition to the inherently poor spatiotemporal resolution (on the order of minutes and hundreds of microns) and the sensitivity loss from derivatization, direct detection of GABA is further limited by its being about 20-fold less abundant than glutamate?

While genetically encoded fluorescent sensors for calcium ${ }^{10,11}$ have been optimized for imaging neural activity, and sensors for the excitatory neurotransmitter glutamate ${ }^{12}$ have been developed, methods for directly imaging GABAergic signaling are lacking. Recently, a semi-synthetic fluorescent sensor for GABA was reported ${ }^{13}$, based on the attachment of organic fluorophores to the $\mathrm{GABA}_{\mathrm{B}}$ receptor. However, its utility in neuronal systems is limited, since it requires the addition of two separate small molecules and its affinity for GABA is extremely weak ( $\sim 400$ 
$\mu \mathrm{M})$. Furthermore, over-expression of GABA receptors could disrupt multiple aspects of cellular signaling, and similarly, hetero-oligomerization with endogenous receptor pools could degrade sensor functionality. Alternatively, inhibitory neurotransmission can be indirectly inferred from intracellular chloride concentration $\left[\mathrm{Cl}^{-}\right]_{\mathrm{i}}$, measurable with Clomeleon and improved variants ${ }^{14}$. However, chloride sensors have poor biophysical performance; $\left[\mathrm{Cl}^{-}\right]_{\mathrm{i}}$ does not change much during inhibition; the sensors conflate the activities of all ligand-gated (GABA, glycine) and voltage-gated $\mathrm{Cl}^{-}$channels; and $\mathrm{GABA}_{\mathrm{B}}$ receptors are $\mathrm{G}_{\mathrm{i} / \mathrm{o}}$-coupled receptors that do not directly affect $\left[\mathrm{Cl}^{-}\right]_{i}$. Thus, direct detection is preferable for inference of GABAergic signaling in general, and is required for mechanistic studies of synaptic input-output transformations, as has been done recently with glutamate release and presynaptic $\mathrm{Ca}^{2+}$ imaging ${ }^{15}$.

We previously reported a method for developing genetically encoded fluorescent sensors with excellent ligand-binding specificity, affinity, and kinetics using bacterial periplasmic binding proteins (PBPs) ${ }^{16}$. This approach to sensor development couples the Venus flytrap-like conformational change that occurs upon ligand binding to changes in the fluorescence of an inserted circularly permuted fluorescent protein. We previously used this technique to develop a glutamate sensor ${ }^{12}$ for in vivo imaging. Here we report similar development of an intensity-based GABA Sensing Fluorescence Reporter (iGABASnFR) and illustrate its utility for observing GABA uptake into mitochondria, and GABA release in cultured neurons and acute mouse brain slice. In vivo, we observed bulk GABA transients in mouse visual cortex, and GABA release synchronized with inter-ictal spikes in a mouse model of epilepsy. Finally, in zebrafish we used iGABASnFR to correlate GABAergic signals across the cerebellum to motor output.

\section{Results}

\section{Sensor engineering}

There are many potential scaffolds for developing a GABA sensor. We deemed the GABAA and $\mathrm{GABA}_{\mathrm{B}}$ receptors inappropriate since the GABA-binding sites of the former are at proteinprotein interfaces $^{2}$, without an obvious route for coupling GABA-dependent conformational changes to effects on a fused fluorescent protein, and the latter is difficult to express heterologously ${ }^{17}$. Furthermore, as discussed above, over-expression of these receptors could alter cell and sensor function in many ways. In theory, one could redesign the binding site of an existing sensor (e.g. iGluSnFR) to make it specific for GABA. Our attempts to do so failed (data not shown).

GABA is a prominent signaling molecule in the plant-soil interface; in fact, it was first isolated from potato tubers ${ }^{18}$. Agrobacterium tumefaciens is a plant pathogen that expresses two periplasmic GABA-binding proteins. Atu2422 binds GABA and other small amino acids ${ }^{19}$. While we were able to develop a sensor from this scaffold, it has very weak (high- $\mu \mathrm{M})$ affinity for GABA, and orders-of-magnitude tighter affinity (low- $\mu \mathrm{M})$ for alanine and glycine (Supp. Fig. S1). Its weak, nonspecific response to GABA makes it an inappropriate sensor scaffold. Atu4243 has more stringent specificity and tighter affinity for GABA $(9 \mu \mathrm{M})^{20}$. Unfortunately, Atu4243 with either cpGFP or cp-superfolderGFP (cpSFGFP) inserted - at locations that we planned to optimize into sensors - did not translocate to the membrane surface in cultured cells when cloned into the pDisplay vector (Invitrogen) (Supp. Fig. S2) and was abandoned as a scaffold. 
Prior to the structures of Atu2422 and Atu4243 being published, a strain of Pseudomonas fluorescens (ATCC \#BAA1781) was reported to rapidly uptake $\mathrm{GABA}^{21}$, presumably through a high-affinity $\mathrm{PBP} /$ transporter. We received a culture of this strain (CNG89; a gift from Dr. Catherine Guthrie) and sequenced its genome. We identified "Pf622" as a homologue of Atu4243 (Supp. Fig. S3) and cloned it by PCR into the pRSET vector (Invitrogen) for heterologous expression in Escherichia coli. Its affinity was not in the low-nM range as expected from the original publication ${ }^{21}$, but rather $130 \mu \mathrm{M}$ as determined by isothermal titration calorimetry (Supp. Fig. S4a). (The calculated molecular weight of Pf622 is $37 \mathrm{kDa}$, and $35 \mathrm{kDa}$ after cleavage of the predicted periplasmic leader peptide. This is smaller than the apparent 42 $\mathrm{kDa}$ determined by SDS-PAGE for the presumed high-affinity GABA-PBP isolated from CNG8922.) A fluorescent allosteric signal transducer ${ }^{23}$, introduced by coupling an environmentally sensitive fluorophore, JF- $585^{24}$, via maleimide-thiol chemistry to a cysteine mutation at a residue in the hinge of the protein (V278C), resulted in a hybrid sensor with 240 $\mu \mathrm{M}$ affinity for GABA (Supp. Fig. S5). Stopped-flow kinetic analysis revealed atypical kinetics. Instead of a single exponential rise in fluorescence upon mixing of protein and GABA, the data is best fit by a double exponential (Supp. Fig. S6a,b), suggesting multiple steps link GABA binding to fluorescence change. Regardless, the rise time for Pf622.V278C-JF585 binding GABA is longer than it is for iGluSnFR binding glutamate (Supp. Fig. S6c).

Pf622 with cpGFP or cpSFGFP inserted at any of several sites translocated to the membrane when cloned into pDisplay and expressed in mammalian cells (Supp. Fig. S7). The improved expression and photostability observed by the substitution of cpSFGFP for cpGFP in iGluSnFR ${ }^{25}$ led us to use the former in this work. Based on its homology to Atu4243, we expected insertion of cpSFGFP after residue 276 of Pf622 (sequence info in Supp. Fig. S8) to yield a good sensor after optimization of the residues linking the binding protein and the FP. Linker optimization yielded a version of the sensor (L1-LA/L2-AN) for which we solved a crystal structure in the unliganded/open state (Supp. Fig. S9). In conjunction with its homology to Atu4243, for which both the unliganded/open and bound/closed structures are available ${ }^{20}$, we tested mutations to the hinge region of Pf622 that we expected to allosterically modulate affinity ${ }^{26}$. Hinge mutation F101L greatly tightened affinity (Supp. Fig. S10). Mutation of a residue in Pf622 (N260A) that potentially interacted with cpSFGFP, and was also a potential glycosylation site, substantially increased brightness in HEK cells (Supp. Fig. S11). Re-optimization of the linkers (L1-LAQVR, L2-AN) plus a mutation in the circularly permutated strand of SFGFP (F145W), resulted in a variant, called iGABASnFR, worthy of further characterization. iGABASnFR is Pf622 with cpSFGFP inserted after residue 276, with optimized linkers (L1-LAQVR, L2-AN), a hinge mutation (F101L), an interface/expression mutation (N260A), and an additional GFP mutation (F145W).

\section{In vitro characterization}

Purified iGABASnFR shows a maximum $\Delta \mathrm{F} / \mathrm{F}$ of $\sim 2.5$ and a $K_{\mathrm{d}}$ for GABA of $\sim 9 \mu \mathrm{M}$ (Supp. Fig. S12a). It shows no affinity for other amino acids except very weak affinity for glycine (500 $\mu \mathrm{M})$, alanine $(830 \mu \mathrm{M})$, and histidine $(2.4 \mathrm{mM})$ (Supp. Fig. 12b). It has no affinity for similar four-carbon metabolites fumarate, malate, oxaloacetate, nor succinate (data not shown). While we characterized iGABASnFR in various cellular systems, we made additional efforts to increase its affinity and $\triangle \mathrm{F} / \mathrm{F}$. Mutation of a GABA-binding pocket residue to its Atu4243 homologue, F102Y, had minimal effect on GABA affinity and decreased $\Delta \mathrm{F} / \mathrm{F}$. Meanwhile, 
mutation $\mathrm{Y} 137 \mathrm{~L}$ increased $\Delta \mathrm{F} / \mathrm{F}$, at the cost of poor expression. The double mutant F102Y.Y137L expressed well, exhibited higher $\Delta \mathrm{F} / \mathrm{F}$ (3.5 vs. 2.5 for iGABASnFR) but weaker affinity (70 $\mu \mathrm{M}$ vs. $9 \mu \mathrm{M}$ for iGABASnFR; Supp. Fig. S12a). Addition of F102G to iGABASnFR (without changes to Y137) also showed higher $\Delta \mathrm{F} / \mathrm{F}(4.5)$ and affinity of $50 \mu \mathrm{M}$. These three variants were further characterized.

We first profiled the variants for binding to GABAergic drugs (Supp. Fig. S12c-f). Baclofen ( $\beta$ (4-chlorophenyl)-GABA) affected all three iGABASnFR variants tested (as well as the negative control cpSFGFP, Supp. Fig. S12c), whereas vigabatrin ( $\gamma$-vinyl-GABA, Sabril®) bound to only the F102G variant. Importantly, most drugs showed much weaker binding to the sensors than GABA, allowing them to be used in conjunction with iGABASnFR imaging.

\section{GABA processing in mitochondria}

After being transported into mitochondria (by an as yet unidentified transporter ${ }^{27}$ ), GABA is degraded by GABA transaminase (GABA-T). Inhibition of GABA-T elevates synaptic levels of GABA $^{28}$ and, of clinical significance, the GABA-T inhibitor vigabatrin is a currently prescribed antiepileptic $^{29}$. We transfected cultured prostate cancer cells (LNCaP) with iGABASnFR.F102Y.Y137L (which doesn't bind vigabatrin; Supp. Fig. S12f), fused to an Nterminal mitochondrial matrix targeting motif from Cox8a (mito-iGABASnFR.F102Y.Y137L). Basal fluorescence in these cells was very low (Supp. Fig. S13), consistent with the observation that these cells contain little $\mathrm{GABA}^{30}$. In the presence of exogenously applied GABA, robust fluorescence was observed within the mitochondria as expected (Fig. 1a). Treatment with varying amounts of GABA increased fluorescence (Fig. 1b, Supp. Fig. S13), reaching equilibrium after 48 hours, a time-course presumably reflecting both increasing mitoiGABASnFR expression and continued GABA uptake. The [GABA] dependence of mitochondria-localized iGABASnFR is approximately linear (Fig. 1c). Vigabatrin treatment tends to increase iGABASnFR fluorescence in the mitochondria (Supp. Fig. S14). To promote endogenous GABA synthesis, LNCaP cells were transfected with a plasmid encoding glutamic acid decarboxylase 1 (GAD1, a.k.a. GAD67). GAD1 expression increased the fluorescence of mito-iGABASnFR.F102Y.Y137L within the first and subsequent time-points (50 hours posttransfection) (Fig. 1d).

\section{Neuronal culture characterization}

HEK cells transfected with iGABASnFR variants cloned into a modified version of pDisplay lacking the HA tag (pMinDis) ${ }^{12}$ showed good membrane localization (Supp. Fig. S11). Primary culture of hippocampal neurons from P0 newborn rats infected with AAV2/1.

hSynapsin 1.iGABASnFR showed good membrane localization and brightness at $14 \mathrm{DIV}$, but the + F102G and + F102Y.Y137L variants were noticeably dimmer, and showed accumulation in the endoplasmic reticulum (Supp. Fig. S15).

Electrical field stimulation $(50 \mathrm{~Hz})$ of the cultured neurons produced fluorescence changes from single stimulus-evoked action potentials and increased until plateauing after 40 AP (Fig. 2a-c). The response was similar to that of SF-iGluSnFR.A184V 25 (Fig. 2d), except that the magnitude of response was ten-fold lower and more localized (Supp. Fig S16), consistent with GABAergic neurons representing a smaller fraction $(\sim 10 \%)$ of the total neuronal population in hippocampal culture $^{31}$. 


\section{Hippocampal acute hippocampal slice}

To confirm that iGABASnFR remains functional in organized brain tissue, we tested iGABASnFR in hippocampal acute slices prepared from mice with iGABASnFR expressed primarily in pyramidal cells through in vivo AAV injection into the hippocampal CA1 region (see Supp. Methods). Although it was challenging to reliably record GABA release with iGABASnFR at the level of individual activated synapses (not shown), as reported previously with iGluSnFR ${ }^{15}$, the sensor displayed excellent $\mathrm{S} / \mathrm{N}$ ratio in response to paired pulse extracellular field stimulation of the Schaffer Collateral pathway, a protocol used widely in synaptic physiology experiments. iGABASnFR responses to extracellular GABA transients could be recorded from apical dendritic segments as short as $10-15 \mu \mathrm{m}$ traced from the CA1 pyramidal cell body (Supp. Fig. S17). Increasing the concentration of extracellular calcium increased the amplitude of fluorescence, consistent with changes in pre-synaptic release probability $^{32}$. It is important to note the value of a spatially resolvable readout of synaptic GABA release given the highly organized and cell type specific nature of GABAergic innervation onto pyramidal cells ${ }^{33}$. Imaging of GABA transients in different cellular compartments enables a high-throughput method to study GABA release from different populations of hippocampal interneurons. Combined with patch-clamp electrophysiology and $\mathrm{Ca}^{2+}$ imaging with red-shifted indicators, iGABASnFR could provide a tool for directly studying the local signaling requirements underlying important physiological phenomena such as depolarization induced suppression of inhibition ${ }^{34}$.

\section{Volume detection of GABA in mouse visual cortex}

Tonic GABA release has recently become appreciated as a mechanism for fine-tuning of circuit function $^{35}$, such as supporting context-dependent behavior in a morphologically fixed cortical circuit $^{36}$. In the visual cortex, GABAergic inhibition ${ }^{37}$ and disinhibition ${ }^{38}$ contribute to contextdependent processing and can enhance the distinctness of sensory responses. Hypnotic effects of volatile anesthetics have been associated with GABAergic transmission ${ }^{39}$. However, depression of cortical activity by anesthetics is accompanied by decreases in both glutamatergic and GABAergic synaptic transmission, with stronger effects on glutamate release ${ }^{40,41}$.

To directly monitor cortical GABA in a time- and depth-resolved manner, we injected AAV2/1. hSynapsin 1.iGABASnFR into mouse primary visual cortex (V1) and measured fluorescence (with $1030 \mathrm{~nm}$ excitation) in a $150 \mu \mathrm{m} \times 150 \mu \mathrm{m}$ x $450 \mu \mathrm{m}$ deep volume over the course of 40 minutes. After 8 minutes, isoflurane anesthesia was administered, which is expected to suppress inhibition $^{41}$. Fluorescence decreased $20 \%$ during anesthesia treatment and slowly returned to baseline during recovery (Supp. Fig. S18).

In vivo mouse model of epilepsy

Having established that iGABASnFR robustly detects large-scale release events, we then tested it in small-scale, high-resolution, 2-photon neuropil imaging experiments. Focal neocortical epilepsy is typically accompanied by abnormal background electrocorticogram (ECoG) activity, including frequent inter-ictal spikes (occurring between seizures), which are dominated by GABAergic activity ${ }^{42}$. How seizures intermittently arise from this background is poorly understood. Numerous mechanisms have been proposed, although the strongest evidence supports a failure of feed-forward inhibition ${ }^{43-46}$. This could, in principle, arise because 
interneurons enter a state of depolarization block in the face of over-excitation and fail to release $\mathrm{GABA}^{47,48}$, or because shifts in the chloride reversal potential in principal cells resulting from intense GABA release render $\mathrm{GABA}_{\mathrm{A}}$ receptor-mediated inhibition ineffective ${ }^{49-52}$. These hypotheses are not mutually exclusive, but important insights could be provided by imaging extracellular GABA during inter-ictal spiking and seizures. If extracellular GABA transients collapse rapidly at the onset of seizures, this would argue for depolarization block of interneurons, or another mechanism by which interneurons fail to be recruited or release GABA.

AAV2/1.hSynapsin1.iGABASnFR (or one of the two other variants) was injected into layer 2/3 of mouse V1 (Fig. 3a-d). Intracortical pilocarpine injection elicited focal epileptiform activity lasting up to 60 minutes ${ }^{53}$. This consisted of periods of inter-ictal spiking alternating with polyspikes and ECoG seizures lasting up to 1-2 minutes. During inter-ictal spiking, mice typically did not manifest abnormal motor behavior, but focal seizures were sometimes accompanied by pupil dilation and increased locomotion ${ }^{54}$. iGABASnFR fluorescence reliably showed transients time-locked to inter-ictal spikes (Fig. 3e-g). Comparing across the different sensors, iGABASnFR and iGABASnFR.F102G gave the largest fluorescence responses, while iGABASnFR.F102Y.Y137L gave lower $\Delta \mathrm{F} / \mathrm{F}$. The non-GABA-binding iGABASnFR.R205A, a negative control (Supp. Fig. S12), was unresponsive (Fig. 3h), indicating that the observed fluorescence traces indeed correspond to GABA transients.

During periods where stereotyped polyspikes were observed, fluorescence peaks separated by $<1$ sec could be resolved with iGABASnFR.F102G (Fig. 4a,b). Discrete peaks coinciding with lowfrequency ECoG components could still be seen during focal seizures lasting up to 1 minute (Fig. 4c). We observed a gradual decrease in fluorescence during seizures, with a slow recovery during intervals of ECoG silence in between seizures (Fig. 4c). This is consistent with the $\mathrm{pH}$ sensitivity of the GABA sensors (Supp. Fig. S19): a decrease in extracellular pH during seizures has been abundantly documented, and possibly factors into seizure termination ${ }^{55}$. The gradual increase in fluorescence between seizures is consistent with a return to normal $\mathrm{pH}$ over several seconds. Importantly, the slow acidification is easily separable from the GABA signals.

\section{GABA in zebrafish cerebellum}

As a final demonstration of the in vivo utility of iGABASnFR, we observed GABA transients in Danio rerio (zebrafish) cerebellum using light-sheet imaging in a fictive model of swimming ${ }^{56,57}$. In zebrafish, fictive swimming activity triggers robust activation of GABAergic Purkinje cells in the cerebellum ${ }^{58}$. We generated a transgenic zebrafish expressing the iGABASnFR.F102Y.Y137L variant under the elavl3/HuC pan-neuronal promoter (Fig. 5a). While the larval fish is paralyzed, and trying to swim in response to forward optic flow, its motor output to the tail muscles is captured by implanted electrodes, so that forward swimming movements can be recorded simultaneously with fluorescence imaging, with minimal motion artifacts (Fig. 5b). Changes in fluorescence within a cerebellar neuropil region onto which GABAergic Purkinje cells project ${ }^{59}$ is clear to the eye (Supp. Movie 1). Changes in fluorescence peaked roughly $200 \mathrm{msec}$ after the onset of swimming (Fig. 5c), with this region of the cerebellum producing upwards of $8 \%$ changes in fluorescence. This result indicates that iGABASnFR can reliably detect GABA transmission that are potentially critical for the motor control of zebrafish.

\section{Discussion}


To understand neural circuit function, numerous specific inputs onto neurons must be disentangled from their integrated output. Current sensor technologies, namely iGluSnFR ${ }^{12,25}$, GCaMP6 ${ }^{11}$ and jGCaMP7 (unpublished), and jRGECO ${ }^{10}$ are sensitive enough to allow reliable detection of excitatory synaptic transmission, excitatory post-synaptic currents, and action potentials. Indicators for inhibitory synaptic transmission and inhibitory post-synaptic currents (IPSCs) have lagged far behind. iGABASnFR offers the best performance of existing GABA indicators, and is the only one appropriate for in vivo use. Calcium and glutamate sensors have been iteratively optimized, both to improve overall performance and to match to precise requirements of specific settings. We expect further development of iGABASnFR, with altered affinity, kinetics, and improved signal-to-noise ratio. Different colors of iGABASnFR could also be developed, allowing simultaneous imaging in orthogonal chromatic channels, as has been done with iGluSnFR ${ }^{25,60}$.

We hope that the demonstrated use of iGABASnFR in models of epilepsy and in the cerebellum of fictively behaving zebrafish will facilitate a better understanding of the role of GABA in various circuits, during development ${ }^{61}$, and in disease states. Aberrations in GABAergic signaling have been demonstrated in Alzheimer's disease ${ }^{62}$, Parkinson's ${ }^{63}$, Huntington' ${ }^{64}$, schizophrenia $^{65}$, and autism/ Rett syndrome ${ }^{66}$. iGABASnFR imaging in appropriate animal models will facilitate greater understanding of these mechanisms. iGABASnFR could also allow easy screening ${ }^{67}$ of candidate transporters such as the vertebrate mitochondrial GABA transporter ${ }^{27}$. Applications outside of neuroscience are also possible, such as separating the critical roles of GABA in plant metabolism and signaling ${ }^{68}$.

\section{Acknowledgements}

We would like to thank Catherine S. Nicholson-Guthrie (Indiana University) for the gift of the Pseudomonas fluorescens strain CNG89. David Stern, Andy Lemire and Damian Kao for helping sequence the genome of CNG89. Deepika Walpita for rat neuronal culture. And John Macklin \& Ronak Patel for collecting 2-photon spectra.

YS, ML and DMK are supported by the Medical Research Council and Wellcome Trust. VM is supported by Epilepsy Research UK. 


\section{References}

1. Ben-Ari, Y., Gaiarsa, J.-L., Tyzio, R. \& Khazipov, R. GABA: a pioneer transmitter that excites immature neurons and generates primitive oscillations. Physiol. Rev. 87, 12151284 (2007).

2. Fritschy, J.-M. \& Panzanelli, P. GABAA receptors and plasticity of inhibitory neurotransmission in the central nervous system. Eur. J. Neurosci. 39, 1845-1865 (2014).

3. Bowery, N. G. et al. International Union of Pharmacology. XXXIII. Mammalian $\gamma-$ aminobutyric acid(B) receptors: structure and function. Pharmacol. Rev. 54, 247-264 (2002).

4. Padgett, C. L. \& Slesinger, P. A. GABAB receptor coupling to G-proteins and ion channels. Adv. Pharmacol. 58, 123-147 (2010).

5. Sodickson, D. L. \& Bean, B. P. GABAB receptor-activated inwardly rectifying potassium current in dissociated hippocampal CA3 neurons. J. Neurosci. 16, 6374-6385 (1996).

6. Bak, L. K., Schousboe, A. \& Waagepetersen, H. S. The glutamate/GABA-glutamine cycle: aspects of transport, neurotransmitter homeostasis and ammonia transfer. $J$. Neurochem. 98, 641-653 (2006).

7. Macdonald, R. L. \& Olsen, R. W. GABAA receptor channels. Annu. Rev. Neurosci. 17, 569-602 (1994).

8. van der Zeyden, M., Oldenziel, W. H., Rea, K., Cremers, T. I. \& Westerink, B. H. Microdialysis of GABA and glutamate: analysis, interpretation and comparison with microsensors. Pharmacol. Biochem. Behav. 90, 135-147 (2008).

9. Ballini, C. et al. Extracellular levels of brain aspartate, glutamate and GABA during an inhibitory avoidance response in the rat. J. Neurochem. 106, 1035-1043 (2008).

10. Dana, H. et al. Sensitive red protein calcium indicators for imaging neural activity. eLife 5, 413 (2016).

11. Chen, T.-W. et al. Ultrasensitive fluorescent proteins for imaging neuronal activity. Nature 499, 295-300 (2013).

12. Marvin, J. S. et al. An optimized fluorescent probe for visualizing glutamate neurotransmission. Nature Methods 10, 162-170 (2013).

13. Masharina, A., Reymond, L., Maurel, D., Umezawa, K. \& Johnsson, K. A fluorescent sensor for GABA and synthetic GABA(B) receptor ligands. J. Am. Chem. Soc. 134, 19026-19034 (2012).

14. Grimley, J. S. et al. Visualization of synaptic inhibition with an optogenetic sensor developed by cell-free protein engineering automation. J. Neurosci. 33, 16297-16309 (2013).

15. Jensen, T. P., Zheng, K., Tyurikova, O., Reynolds, J. P.\& Rusakov, D. A. Monitoring single-synapse glutamate release and presynaptic calcium concentration in organised brain tissue. Cell Calcium 64, 102-108 (2017).

16. Marvin, J. S., Schreiter, E. R., Echevarría, I. M. \& Looger, L. L. A genetically encoded, high-signal-to-noise maltose sensor. Proteins 79, 3025-3036 (2011).

17. Geng, Y. et al. Structure and functional interaction of the extracellular domain of human GABA(B) receptor GBR2. Nature Neurosci. 15, 970-978 (2012). 
18. Steward, F. C., Thompson, J. F. \& Dent, C. E. $\gamma$-Aminobutyric Acid: A Constituent of the Potato Tuber? Science 110, 439-440 (1949).

19. Planamente, S. et al. A conserved mechanism of GABA binding and antagonism is revealed by structure-function analysis of the periplasmic binding protein Atu2422 in Agrobacterium tumefaciens. J. Biol. Chem. 285, 30294-30303 (2010).

20. Planamente, S. et al. Structural basis for selective GABA binding in bacterial pathogens. Molecular Microbiology 86, 1085-1099 (2012).

21. Guthrie, G. D. \& Nicholson-Guthrie, C. S. $\gamma$-Aminobutyric acid uptake by a bacterial system with neurotransmitter binding characteristics. Proc. Natl. Acad. Sci. U.S.A. 86, 7378-7381 (1989).

22. Guthrie, G. D., Nicholson-Guthrie, C. S. \& Leary, H. L., Jr. A Bacterial High-Affinity GABA Binding Protein: Isolation and Characterization. Biochem. Biophys. Res. Comm. 268, 65-68 (2000).

23. Marvin, J. S. et al. The rational design of allosteric interactions in a monomeric protein and its applications to the construction of biosensors. Proc. Natl. Acad. Sci. U.S.A. 94, 4366-4371 (1997).

24. Grimm, J. B. et al. A general method to fine-tune fluorophores for live-cell and in vivo imaging. Nature Methods 14, 987-994 (2017).

25. Marvin, J. S. et al. Stability, affinity and chromatic variants of the glutamate sensor iGluSnFR. bioRxiv (2018). doi:10.1101/235176

26. Marvin, J. S. \& Hellinga, H. W. Manipulation of ligand binding affinity by exploitation of conformational coupling. Nat. Struct. Biol. 8, 795-798 (2001).

27. Ravasz, D. et al. Catabolism of GABA, succinic semialdehyde or $\gamma$-hydroxybutyrate through the GABA shunt impair mitochondrial substrate-level phosphorylation. Neurochem. Internat. 109, 41-53 (2017).

28. Engel, D. et al. Plasticity of rat central inhibitory synapses through GABA metabolism. $J$. Physiol. 535, 473-482 (2001).

29. Brunton, L. L., Chabner, B. \& Knollmann, B. C. Goodman \& Gilman's the pharmacological basis of therapeutics. (2011). New York, McGraw-Hill.

30. Putluri, N. et al. Metabolomic profiling reveals a role for androgen in activating amino acid metabolism and methylation in prostate cancer cells. PLoS ONE 6, e21417 (2011).

31. Benson, D. L., Watkins, F. H., Steward, O. \& Banker, G. Characterization of GABAergic neurons in hippocampal cell cultures. J. Neurocytol. 23, 279-295 (1994).

32. Dodge, F. A. \& Rahamimoff, R. Co-operative action of calcium ions in transmitter release at the neuromuscular junction. J. Physiol. 193, 419-432 (1967).

33. Somogyi, P. \& Klausberger, T. Defined types of cortical interneurone structure space and spike timing in the hippocampus. J. Physiol. 562, 9-26 (2005).

34. Ohno-Shosaku, T. et al. Presynaptic cannabinoid sensitivity is a major determinant of depolarization-induced retrograde suppression at hippocampal synapses. J. Neurosci. 22, 3864-3872 (2002).

35. Farrant, M. \& Nusser, Z. Variations on an inhibitory theme: phasic and tonic activation of GABA(A) receptors. Nat. Rev. Neurosci. 6, 215-229 (2005).

36. Kuchibhotla, K. V. et al. Parallel processing by cortical inhibition enables contextdependent behavior. Nature Neurosci. 20, 62-71 (2017).

37. Adesnik, H., Bruns, W., Taniguchi, H., Huang, Z. J. \& Scanziani, M. A neural circuit for spatial summation in visual cortex. Nature 490, 226-231 (2012). 
38. Zhang, S. et al. Selective attention. Long-range and local circuits for top-down modulation of visual cortex processing. Science 345, 660-665 (2014).

39. Nelson, L. E. et al. The sedative component of anesthesia is mediated by GABA(A) receptors in an endogenous sleep pathway. Nature Neurosci. 5, 979-984 (2002).

40. Westphalen, R. I. \& Hemmings, H. C. Selective depression by general anesthetics of glutamate versus GABA release from isolated cortical nerve terminals. J. Pharmacol. Exp. Ther. 304, 1188-1196 (2003).

41. Ferron, J.-F., Kroeger, D., Chever, O. \& Amzica, F. Cortical inhibition during burst suppression induced with isoflurane anesthesia. J. Neurosci. 29, 9850-9860 (2009).

42. Cohen, I., Navarro, V., Clemenceau, S., Baulac, M. \& Miles, R. On the origin of interictal activity in human temporal lobe epilepsy in vitro. Science 298, 1418-1421 (2002).

43. Trevelyan, A. J., Sussillo, D. \& Yuste, R. Feedforward inhibition contributes to the control of epileptiform propagation speed. J. Neurosci. 27, 3383-3387 (2007).

44. Trevelyan, A. J., Sussillo, D., Watson, B. O. \& Yuste, R. Modular propagation of epileptiform activity: evidence for an inhibitory veto in neocortex. J. Neurosci. 26, 1244712455 (2006).

45. Schevon, C. A. et al. Evidence of an inhibitory restraint of seizure activity in humans. Nature Commun. 3, 1060 (2012).

46. Prince, D. A. \& Wilder, B. J. Control mechanisms in cortical epileptogenic foci. 'Surround' inhibition. Arch. Neurol. 16, 194-202 (1967).

47. Cammarota, M., Losi, G., Chiavegato, A., Zonta, M. \& Carmignoto, G. Fast spiking interneuron control of seizure propagation in a cortical slice model of focal epilepsy. $J$. Physiol. 591, 807-822 (2013).

48. Ziburkus, J., Cressman, J. R., Barreto, E. \& Schiff, S. J. Interneuron and pyramidal cell interplay during in vitro seizure-like events. J. Neurophysiol. 95, 3948-3954 (2006).

49. Pavlov, I., Kaila, K., Kullmann, D. M. \& Miles, R. Cortical inhibition, pH and cell excitability in epilepsy: what are optimal targets for antiepileptic interventions? J. Physiol. 591, 765-774 (2013).

50. Nardou, R. et al. Neuronal chloride accumulation and excitatory GABA underlie aggravation of neonatal epileptiform activities by phenobarbital. Brain 134, 987-1002 (2011).

51. Kaila, K., Lamsa, K., Smirnov, S., Taira, T. \& Voipio, J. Long-lasting GABA-mediated depolarization evoked by high-frequency stimulation in pyramidal neurons of rat hippocampal slice is attributable to a network-driven, bicarbonate-dependent $\mathrm{K}+$ transient. J. Neurosci. 17, 7662-7672 (1997).

52. Staley, K. J., Soldo, B. L. \& Proctor, W. R. Ionic mechanisms of neuronal excitation by inhibitory GABAA receptors. Science 269, 977-981 (1995).

53. Kätzel, D., Nicholson, E., Schorge, S., Walker, M. C. \& Kullmann, D. M. Chemicalgenetic attenuation of focal neocortical seizures. Nature Commun. 5, 3847 (2014).

54. Rossi, L. F., Wykes, R. C., Kullmann, D. M. \& Carandini, M. Focal cortical seizures start as standing waves and propagate respecting homotopic connectivity. Nature Commun. 8, 217 (2017).

55. Ziemann, A. E. et al. Seizure termination by acidosis depends on ASIC1a. Nature Neurosci. 11, 816-822 (2008).

56. Ahrens, M. B. et al. Brain-wide neuronal dynamics during motor adaptation in zebrafish. Nature 485, 471-477 (2012). 
57. Vladimirov, N. et al. Light-sheet functional imaging in fictively behaving zebrafish. Nature Methods 11, 883-884 (2014).

58. Scalise, K., Shimizu, T., Hibi, M. \& Sawtell, N. B. Responses of cerebellar Purkinje cells during fictive optomotor behavior in larval zebrafish. J. Neurophysiol. 116, 2067-2080 (2016).

59. Tanabe, K. et al. Atypical protein kinase $\mathrm{C}$ regulates primary dendrite specification of cerebellar Purkinje cells by localizing Golgi apparatus. J. Neurosci. 30, 16983-16992 (2010).

60. Wu, J. et al. Genetically Encoded Glutamate Indicators with Altered Color and Topology. ACS Chem. Biol. (2018). doi:10.1021/acschembio.7b01085

61. Ben-Ari, Y. The GABA excitatory/inhibitory developmental sequence: a personal journey. Neuroscience 279, 187-219 (2014).

62. Jo, S. et al. GABA from reactive astrocytes impairs memory in mouse models of Alzheimer's disease. Nature Medicine 20, 886-896 (2014).

63. Emir, U. E., Tuite, P. J. \& Öz, G. Elevated pontine and putamenal GABA levels in mildmoderate Parkinson disease detected by 7 tesla proton MRS. PLoS ONE 7, e30918 (2012).

64. Raymond, L. A. et al. Pathophysiology of Huntington's disease: time-dependent alterations in synaptic and receptor function. Neuroscience 198, 252-273 (2011).

65. Simpson, M. D., Slater, P., Deakin, J. F., Royston, M. C. \& Skan, W. J. Reduced GABA uptake sites in the temporal lobe in schizophrenia. Neurosci. Lett. 107, 211-215 (1989).

66. Chao, H.-T. et al. Dysfunction in GABA signalling mediates autism-like stereotypies and Rett syndrome phenotypes. Nature 468, 263-269 (2010).

67. Keller, J. P. \& Looger, L. L. The Oscillating Stimulus Transporter Assay, OSTA: Quantitative Functional Imaging of Transporter Protein Activity in Time and Frequency Domains. Molecular Cell 64, 199-212 (2016).

68. Michaeli, S. \& Fromm, H. Closing the loop on the GABA shunt in plants: are GABA metabolism and signaling entwined? Front. Plant. Sci. 6, 419 (2015). 
Fig. 1. Transport of GABA into the mitochondria is detected by mito-

iGABASnFR.F102Y.Y137L. a) LNCaP cells were transfected with mito-

iGABASnFR.F102Y.Y137L in the presence of $100 \mu \mathrm{M}$ GABA. 24 hours after transfection, cells were incubated with MitoTracker Red. Epifluorescence (60x objective) shows co-localization of the two signals. Left, green channel. Middle, red channel. Right, merged. b) LNCaP cells were transfected with mito-iGABASnFR and the media supplemented with GABA 24 hours posttransfection. Fluorescence was measured 2 hours after treatment, and every 4 hours thereafter. $b$ ) Time course of fluorescence for different GABA treatments $(0,1,10,100,1000$, and 10,000 $\mu \mathrm{M}$ GABA, light grey to black, respectively). c) [GABA] dependence of response was determined by averaging three time points (46, 50, 54 hours) within a single experiment. d) Fluorescence of LNCaP cells transfected with mito-iGABASnFR.F102Y.Y137L and a vector expressing glutamate decarboxylase 1 (GAD1, a.k.a. GAD67) relative to an empty vector control $(n=6)$. Fluorescence was measured beginning 50 hours post-transfection. Black, GAD1; grey, empty vector. For all plots, fluorescence was quantified with thresholding using an automated algorithm (see Methods).

a)

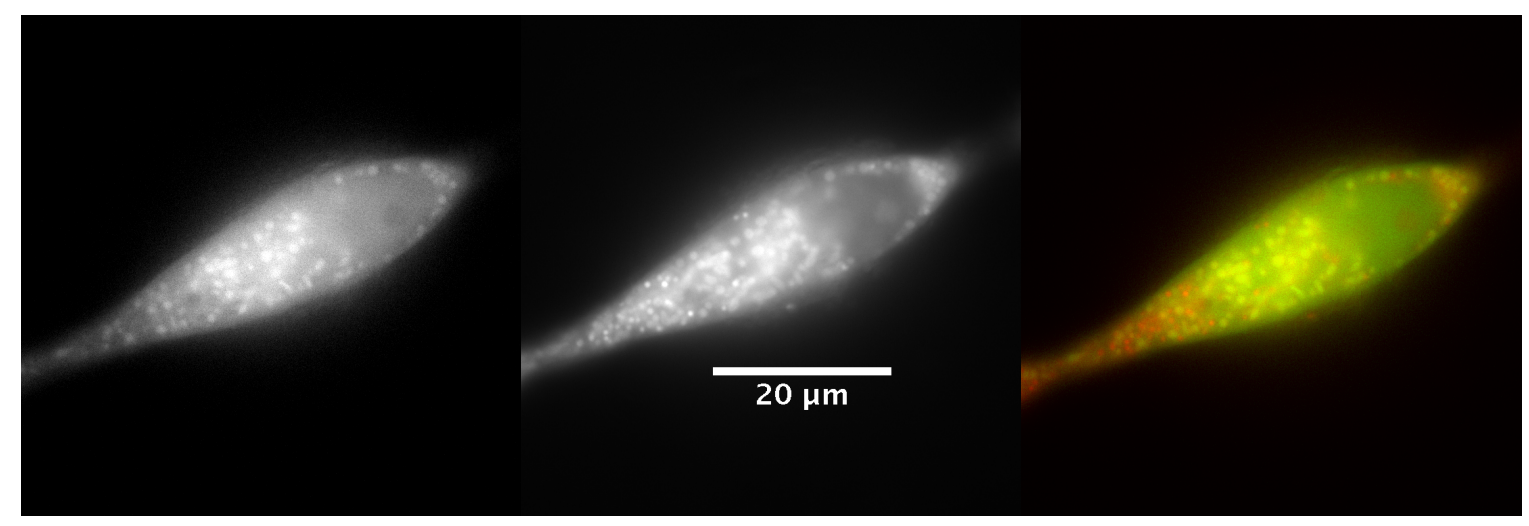


bioRxiv preprint doi: https://doi.org/10.1101/322578; this version posted May 14, 2018. The copyright holder for this preprint (which was not certified by peer review) is the author/funder, who has granted bioRxiv a license to display the preprint in perpetuity. It is made available under aCC-BY-NC-ND 4.0 International license.

b)

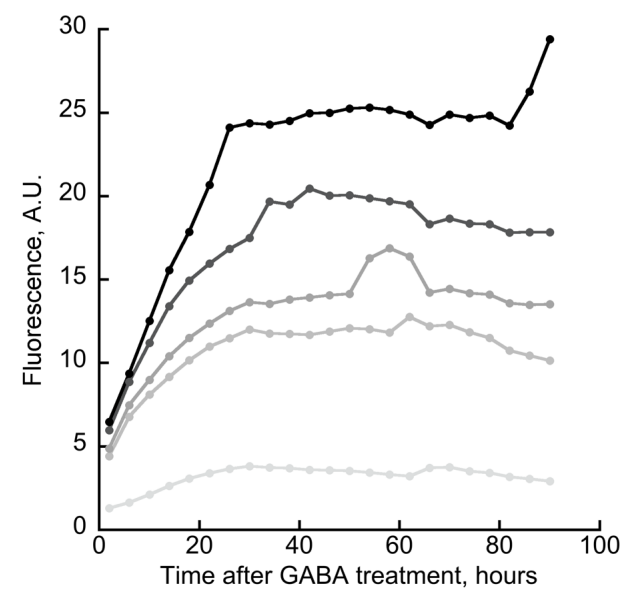

d)

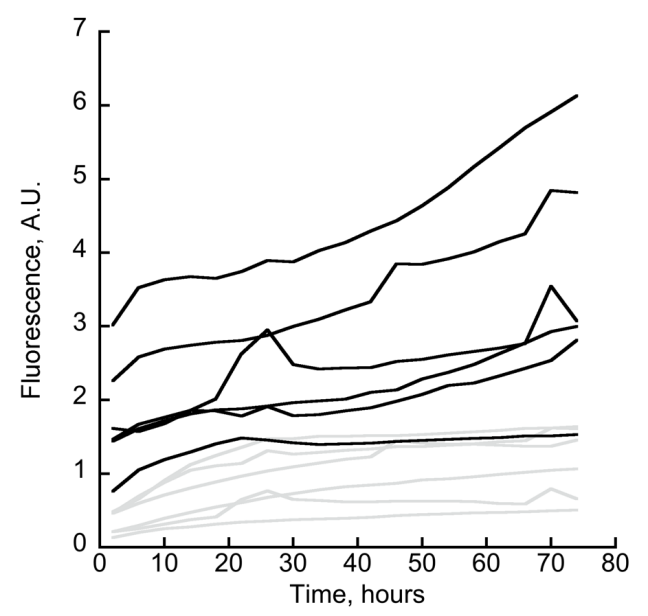

c)

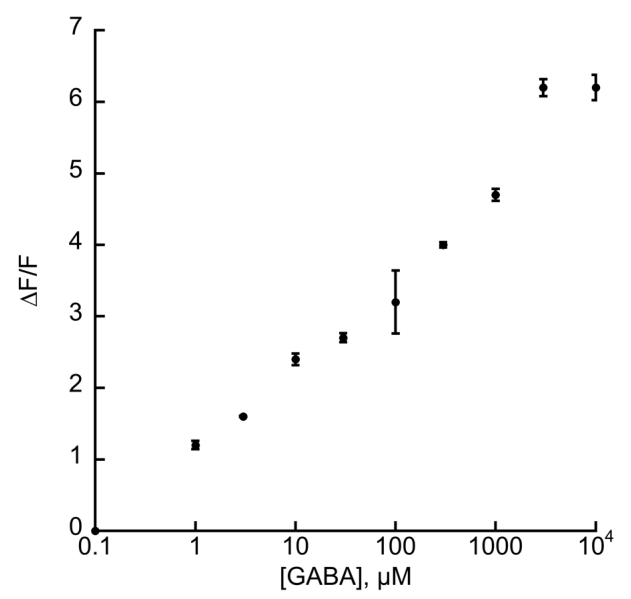


bioRxiv preprint doi: https://doi.org/10.1101/322578; this version posted May 14, 2018. The copyright holder for this preprint (which was not certified by peer review) is the author/funder, who has granted bioRxiv a license to display the preprint in perpetuity. It is made available under aCC-BY-NC-ND 4.0 International license.

Fig. 2. Response of iGABASnFR.F102G in rat hippocampal culture 14 DIV to whole field electrical stimulation. a) Fluorescence image. b) Heat map showing $\Delta \mathrm{F} / \mathrm{F}$ in response to $10 \mathrm{AP}$ stimulation. c) Averaged response (mean \pm std.dev., $n=3$ ) of an ROI selected in a region of maximal $\Delta \mathrm{F} / \mathrm{F}$ to multiple stimuli $(50 \mathrm{~Hz})$. Black to light grey: 1, 2, 5, 10, 20, 40 field stimuli. SF-iGluSnFR.A184V responses performed in side-by-side cultures are in Supp. Fig. S16. Scale bar $20 \mu \mathrm{m}$.

a)

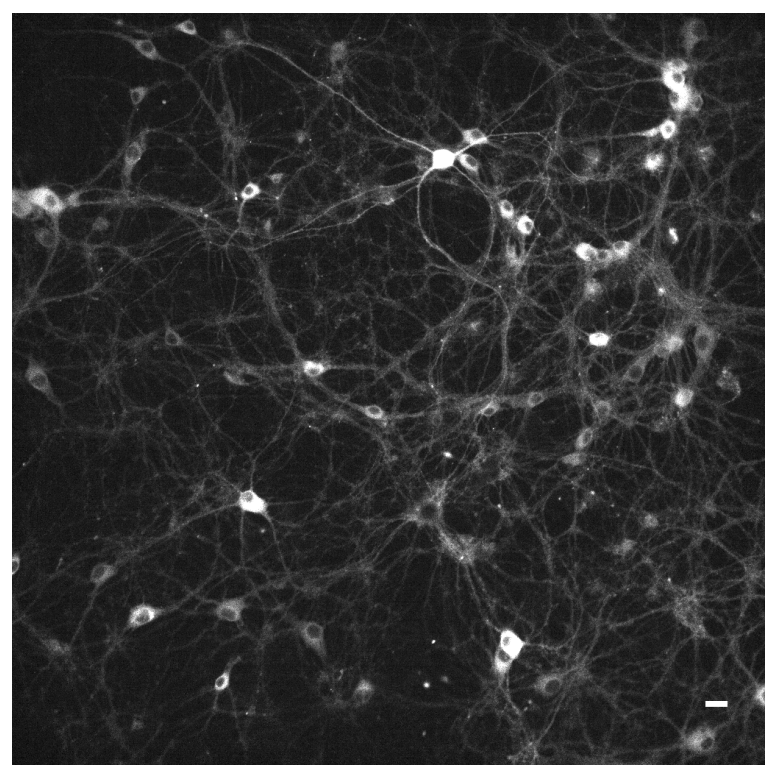

b)

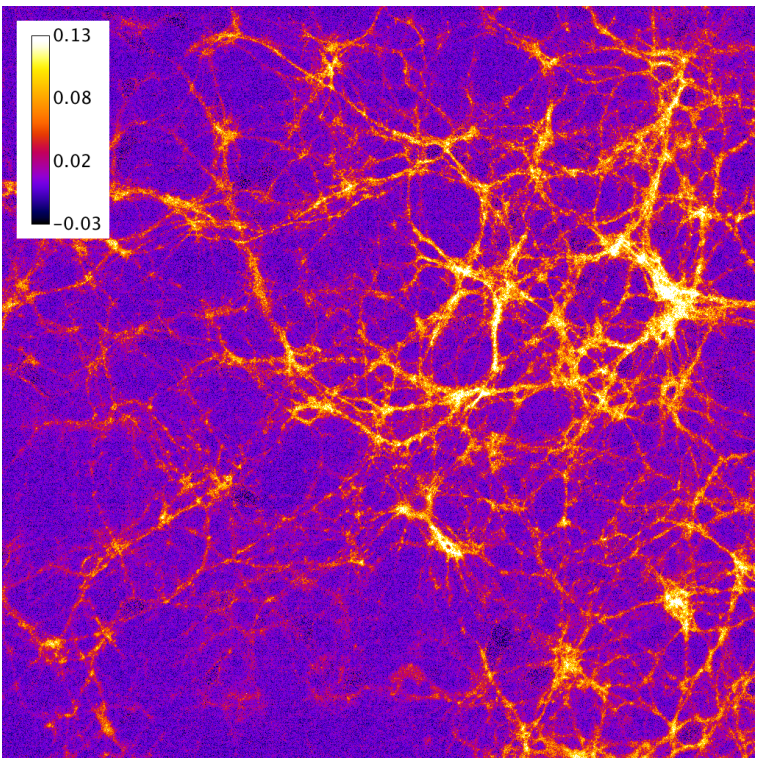

c)

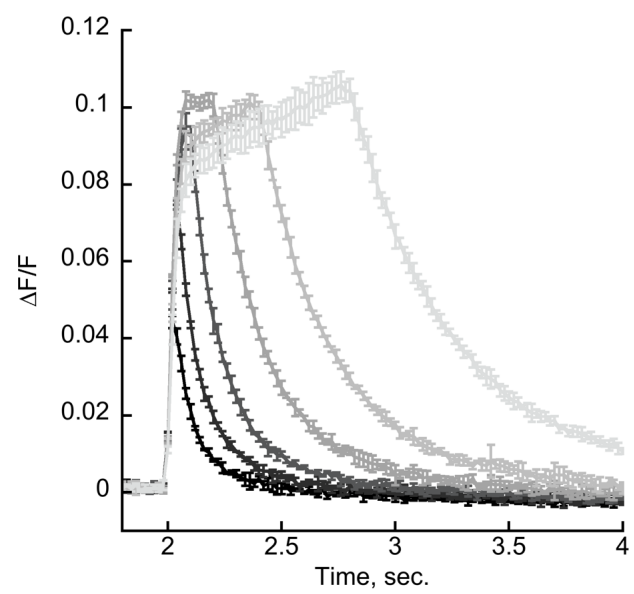


Fig. 3. iGABASnFR response during inter-ictal spiking. a) Experimental design. b) iGABASnFR expression in transduced area imaged after the end of the experiment, using immunofluorescence microscopy. Scale bar: $100 \mu \mathrm{m}$. c) iGABASnFR expression in vivo in cortical pyramidal neuron processes $100 \mu \mathrm{m}$ below the pia. Scale bar: $20 \mu \mathrm{m}$. d) Average iGABASnFR fluorescence image during inter-ictal activity. Scale bar: $50 \mu \mathrm{m}$. e,f) Simultaneous fluorescence (averaged in green square in d) and inter-ictal ECoG. Grey bar indicates the enlargement window in f. g). Timecourse of iGABASnFR fluorescence and inter-ictal ECoG averaged from 68 events in one experiment. h) Comparison of average fluorescence transients obtained with different sensors. 
A AAV2/1.hSyn.Pf622.3B. F101L.N260A.F145W in V1

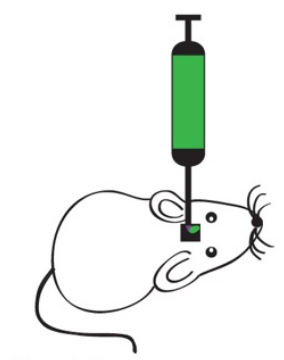

Craniotomy, electrode implantation

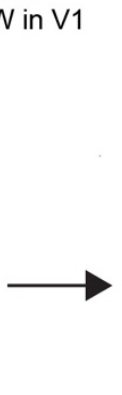

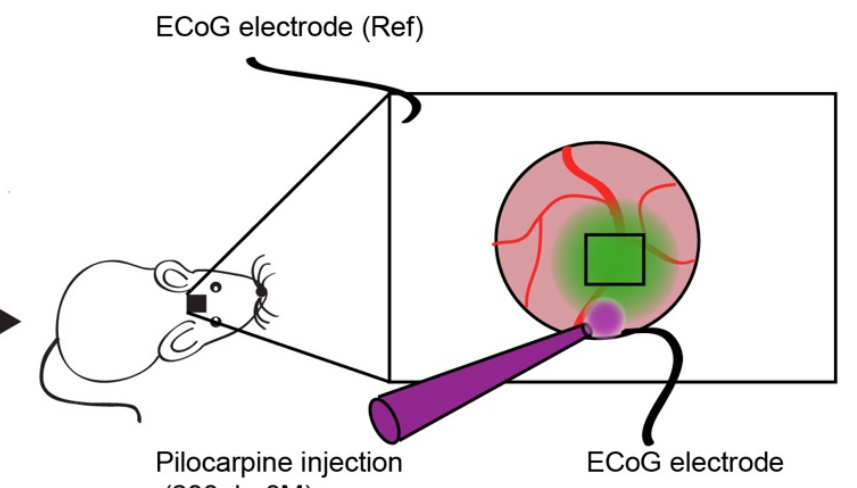

B
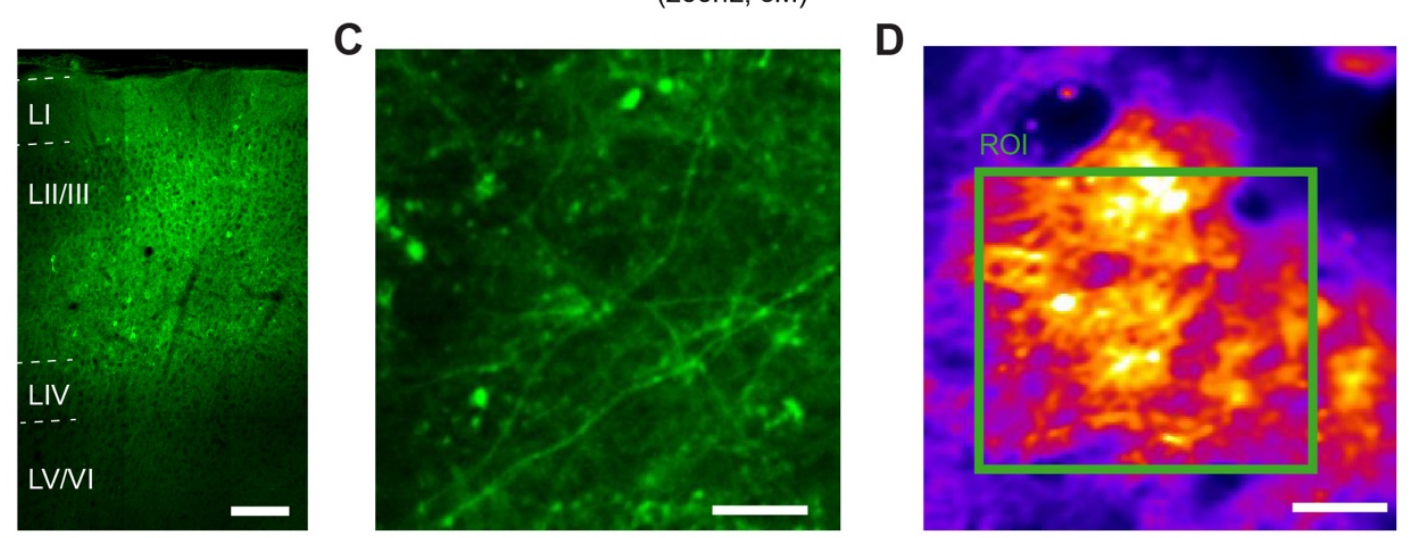

E
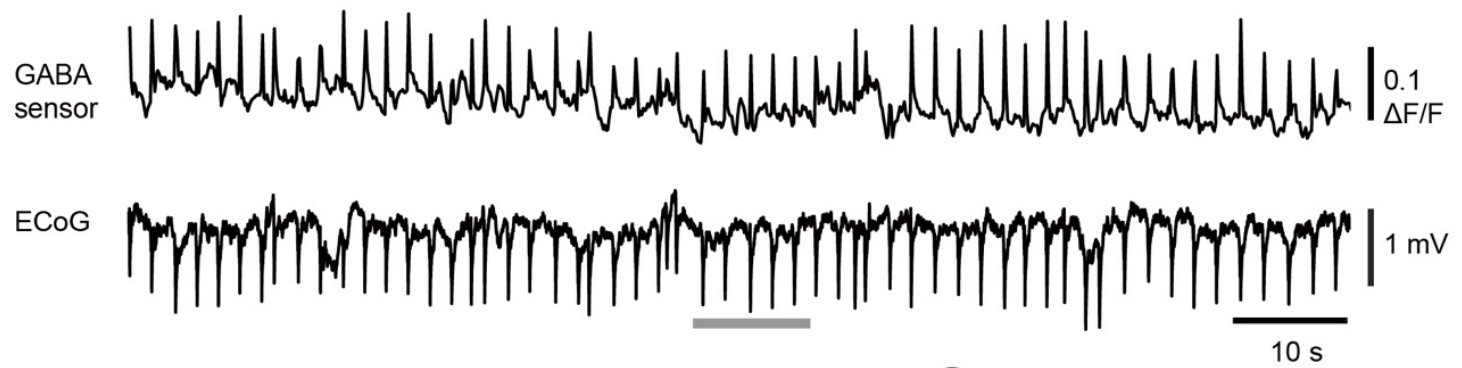

$\mathbf{F}$

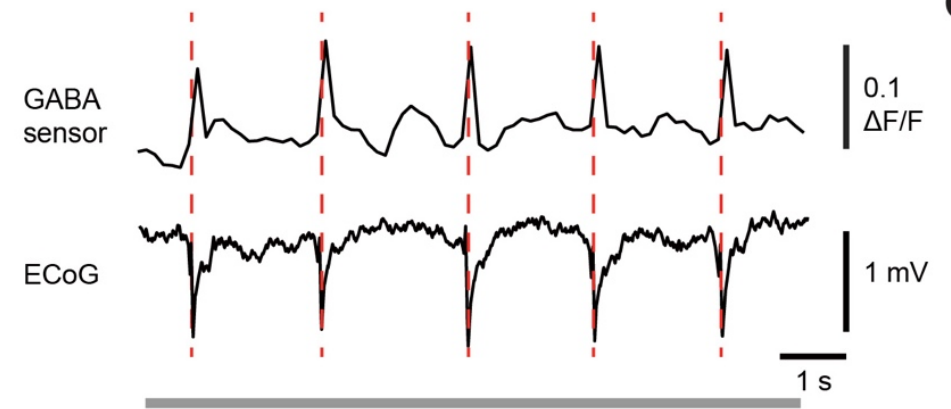

G

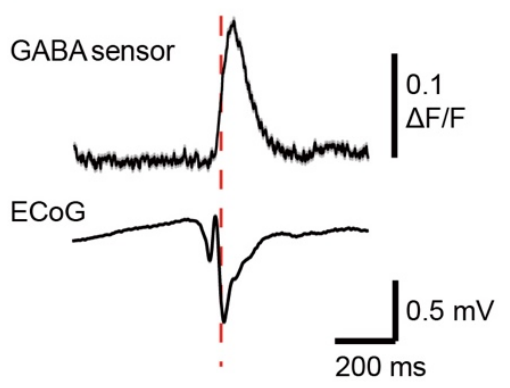

H

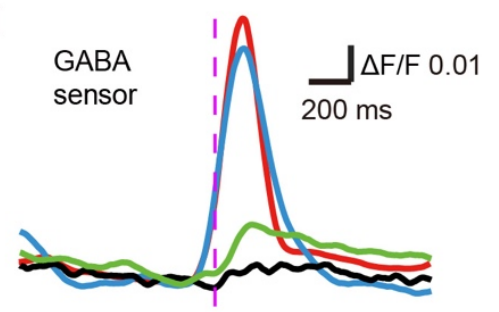

N260A.F145W

N260A.F145W F102G

F101.N260A.F145W 102Y.Y137L

F101 N260A.F145W R205A 
Fig. 4. GABA sensor behavior during polyspikes and seizures. a) Trains of polyspikes (ECoG, bottom) and simultaneous iGABASnFR.F102G fluorescence (top) evoked by pilocarpine injection. b) Average time-course of sensor fluorescence and ECoG during polyspike. c) iGABASnFR.F102G fluorescence during seizures. Note that the envelope of the fluorescence transients gradually decreases during seizures, and then slowly recovers during periods of electrographic silence before the return of seizure activity.

A

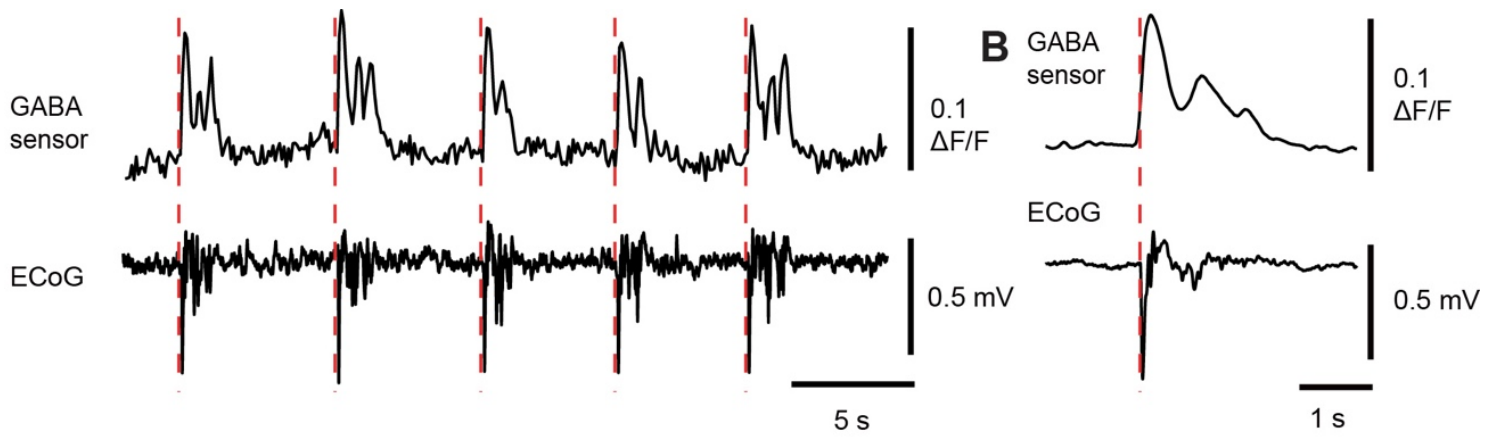

C

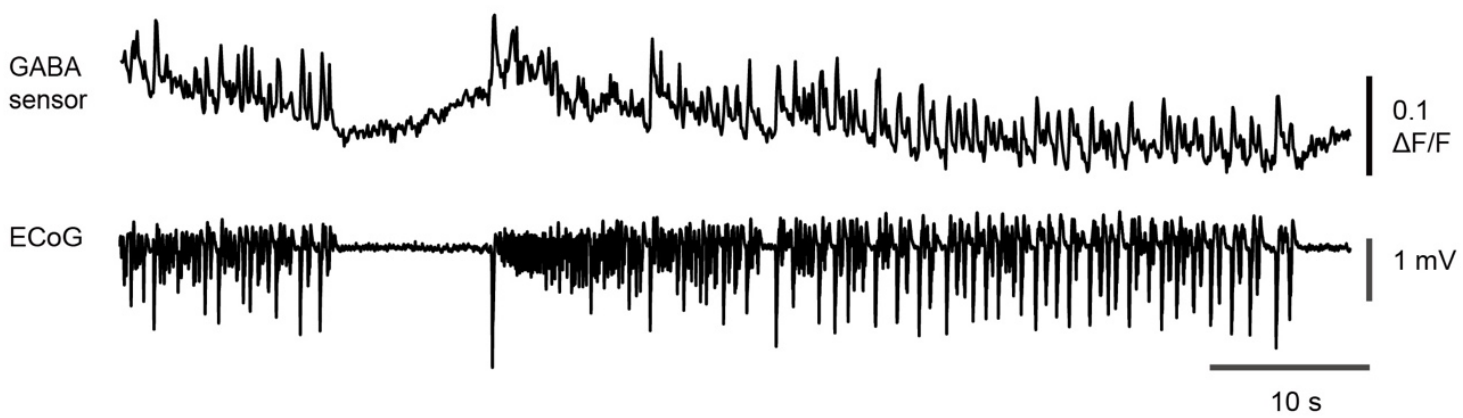


Fig. 5. GABA response seen with iGABASnFR.F102Y.Y137L fluorescence in a fictive model of swimming in zebrafish larvae. See also Supp. Movie 1, in which changes in fluorescence within the region denoted by an asterisk* are obvious. a) Light sheet image of zebrafish cerebellum expressing iGABASnFR.F102Y.Y137L under the elavl3/HuC promoter. b) Motor signals (grey) and fluorescent signals from ROIs indicated in (a). c) Motor signals (left) and fluorescence changes (right) averaged across 5 swim events. Shadows represent s.e.m. across swim events.

a

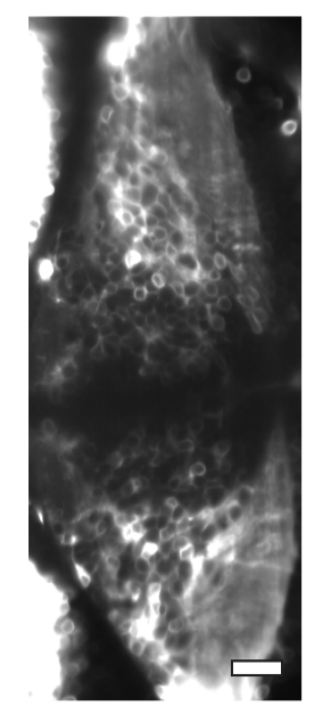

b

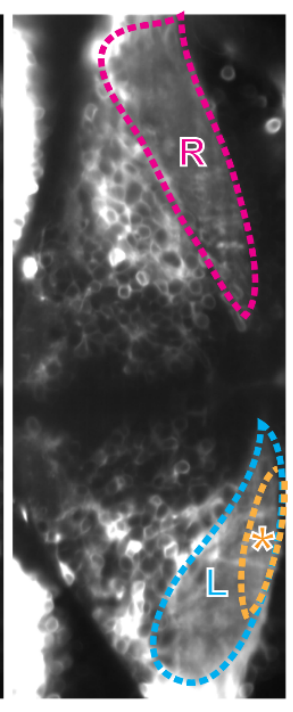

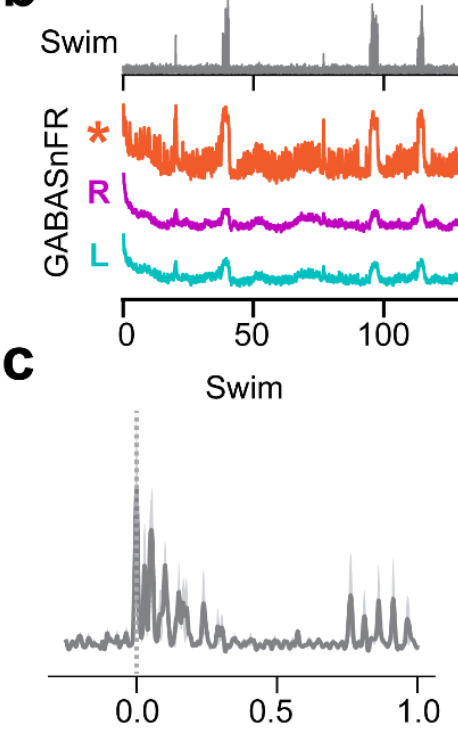

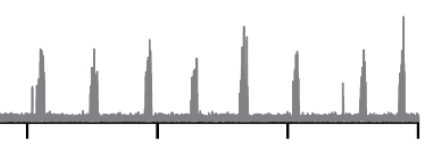

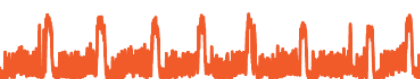

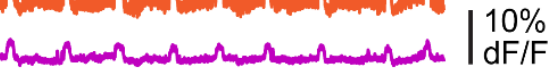

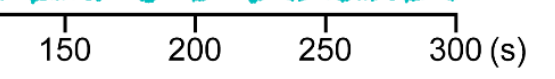

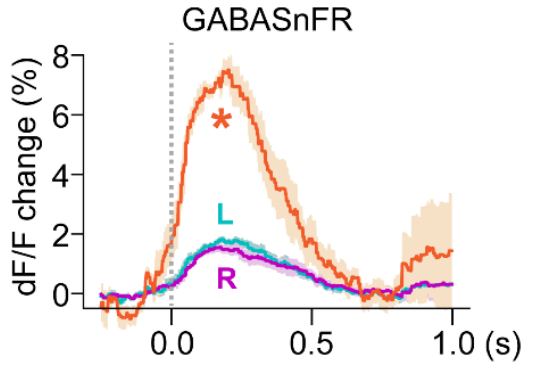

